\title{
Effects of Water Application Rates, Plastic-Mulch, and Staking on Size Arrangements of Mature Green Tomatoes Under Drip Irrigation ${ }^{1}$
}

\author{
Rubén Guadalupe-Luna, Megh R. Goyal, Magaly Cintrón, Luis E. Rivera \\ and María del C. Prieto de López ${ }^{2}$
}

\begin{abstract}
Size arrangements, percentage distribution of fresh harvested mature green tomatoes and vitamin A content were evaluated for plastic mulched-staked (PS), plastic mulched-nonstaked (PNS), non-mulched-staked (NPS), and nonmulched-nonstaked (NPNS) tomatoes under drip irrigation in wet, moist and dry treatments, respectively. Percentage distribution of number of fruits was highest in USDA fruit size class No. 6 for all treatments and subtreatments. For all treatments, the average fruit weight was highest during the second picking in the NPNS, NPS and PNS plots compared to the first picking in the PS plots. The dry treatment gave highest percentage of US grade I tomatoes in the NPNS and PS plots compared to that of the moist treatment in the NPS and the wet treatment in the PNS plots, respectively. The vitamin A content was highest in NPNS plots for wet and moist treatments, and in PS plots for dry treatment. The PS plots in the wet and moist treatments, and NPS plots in the dry treatment gave the lowest values of vitamin A content.
\end{abstract}

\section{INTRODUCTION}

The market for fresh and processed vegetables in Puerto Rico amounts to over 70 million yearly, about $83 \%$ of which is imported. Local demand for vegetable products and fresh vegetables as well as the interest of local industry in marketing fresh vegetables, is increasing.

The climate and soil resources of Puerto Rico are well suited to the growing of a wide variety of vegetables. Several crops may be obtained from the same field every year. For several years the sugar cane industry has been consistently decreasing its production, leaving appreciable acreage of land available for other agricultural uses. Proximity of growing regions to principal local markets and opportunities for export of surplus produce to the winter market in the United States provide further incentives for the development of an efficient fresh vegetable industry.

For the last few years a major research program has been in progress in Puerto Rico for the commercial production of vegetables. Furthermore, the Commonwealth Department of Agriculture has initiated a new agricultural program in which emphasis is being given to the production of

${ }^{1}$ Manuscript submitted to Editorial Board November 6, 1981.

This study was conducted under H326(S-143), Southern Region Research Project"Trickle Irrigation in Humid Regions" and project H-284, "Grading of Vegetables."

${ }^{2}$ Associate Horticulturist, Assistant Agricultural Engineer, Assistant Chemist, Scientific Research Technician, and Research Assistant, respectively, Agricultural Experiment Station, University of Puerto Rico, Mayagüez Campus, Rio Piedras, P.R. 
vegetables for the fresh market and processing. The principal objectives of this program deal with the different aspects of production including evaluation of local and imported varieties of vegetables; the determination of the optimum cultivation practices under our soil and climatic conditions; effects of season of the year on yield and quality; determination of the most effective methods for the control of diseases and insects and other pests; and the economics of production as related to marketing strategies. This research program provides for the study of harvesting, handling, storage and transportation practices in marketing of fresh vegetables in Puerto Rico. This program is supplemented by investigations on grading for measuring variations in quality in comformity to USDA grade standards of locally grown produce.

In the United States almost all agricultural commodities are marketed on the basis of standards established under federal or state laws. Such standards provide the first step in orderly marketing by providing a common language for producers, packers, buyers and consumers. Standardized grades form the basis for prices published in market news reports and are necessary for a meaningful comparison of prices. They enable pooling of products with reimbursements to individual members on an equitable basis; they are necessary as a basis for advertising and provide a basis for financing an industry in which standardized grades are of great importance in establishing values.

To be most useful and meet the needs of food producers and users grades and standards are developed by government agencies working together with trade and consumer groups. A standard which cannot be met by a majority of producers or fails to reflect attributes judged important by consumers is of limited value. However, because of agronomic factors or differences in consumer acceptance of quality, attributes considered important in the United States, may not be applicable to crops grown in Puerto Rico. This is why grading standards developed in the U.S. must be investigated with respect to their applicability in Puerto Rico.

For local vegetable crops to compete successfully with imported vegetables, there must be standardized grades. These are needed for the production of high quality and more uniform produce to supply the local market. Separation of products into various grades and trading on the basis of quality is the greatest stimulus to better methods of production and marketing because grading helps growers to pool their products in cooperative marketing associations in order to share equitably in the season's sales. Quality standards are of great value to farmers and consumers. Any variation in appearance, texture, taste and other characteristics usually make a difference in the selling price of produce; and 
the consumers, who are better informed and more demanding with respect to food quality, generally get greater values by buying graded products. Finally, trading on the basis of quality is the greatest stimulus for improving methods of production and marketing because it helps growers and shippers to correct their mistakes. Over the years much work has been done on vegetable quality and grades, but for the most part this has dealt with vegetables grown and marketed under temperate zone conditions. To what extent USDA grading standards are applicable to vegetables grown in Puerto Rico remains to be determined.

The objectives of this study were to evaluate effects of varying water application rates, plastic mulch and staking on vitamin A content, size arrangements and fruit quality of mature green tomatoes (var. Walter) under drip irrigation.

\section{MATERIALS AND METHODS}

This study was conducted at the Fortuna Substation, located in the semiarid southern coast of Puerto Rico. The soil belongs to the San Antón series with a $\mathrm{pH}$ of 7.9. Maximum, minimum and average temperatures during the growing period were $34.0,16.0$ and $25.0^{\circ} \mathrm{C}$, respectively. The seasonal rainfall and class A pan evaporation were 51 and $551 \mathrm{~mm}$, respectively ${ }^{3}$.

The crop was subjected to three water regimes (wet, moist and dry) based upon readings of tensiometers which were installed at 15, 30 and $45 \mathrm{~cm}$ below the soil surface to control the irrigation scheduling. The main treatments were replicated six times in a randomized split-plot block design (Fig. 1). The subtreatments were plastic mulched-staked (PS), plastic mulched-nonstaked (PNS), nonmulched-staked (NPS) and nonmulched-nonstaked (NPNS). Four-week-old tomato transplants were planted November 13, 1980, $15 \mathrm{~cm}$ from the dual chamber drip line and on the windward side of the drip line. In the subplots (PS, NPS), tomatoes were staked with bamboo-twine on 33, 41 and 51st day after planting. In order to determine the optimum harvest time and the quality ranges, which may be expected at different seasons for local and imported varieties, 50 "mature green" tomatoes were sampled, classified and graded on the 67, 81 and 90th day. The samples from each treatment and subtreatment were evaluated for size, weight, vitamin A content and visible damage according to "USDA-tomatoes shipping point instructions."

\footnotetext{
${ }^{3}$ Trickle Irrigation in Humid Regions-Puerto Rico. Annual report no. 2/PR/H326(S143)/1980-81. October 1, 1980 to September 30, 1981. Agric. Exp. Stn. Univ. P.R., Río Piedras, P.R. Page 150.
} 


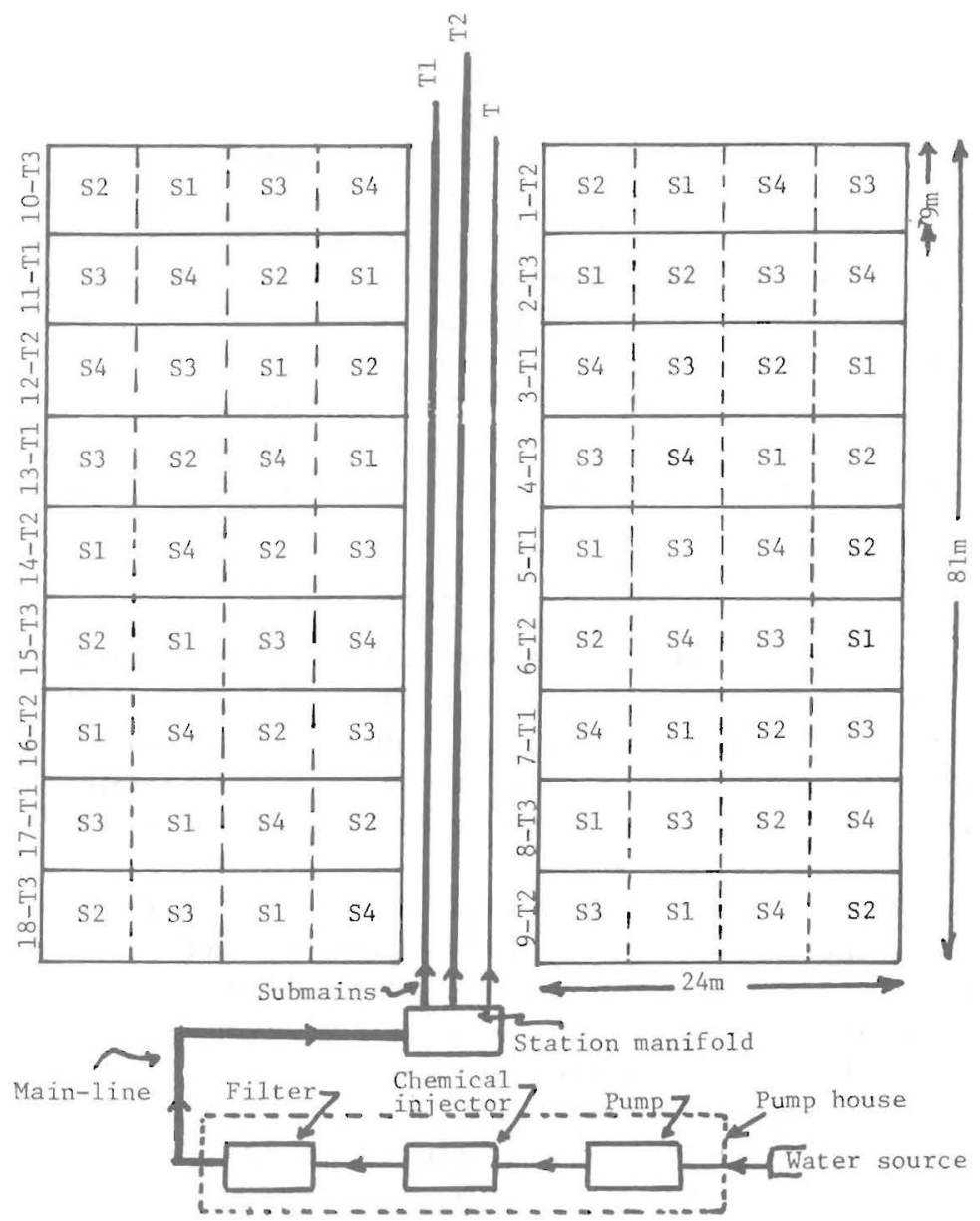

Main treatments

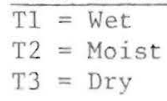

$$
\begin{aligned}
& \text { Subtreatments } \text { No plastic-no stakes (NPNS) } \\
& \text { S1 }=\text { No plastic-stakes (NPS) } \\
& \text { S3 }=\text { Plastic-no stakes(PNS) } \\
& \text { S4 = Plastic-stakes (PS) }
\end{aligned}
$$

Plot size $=24 \times 9 \mathrm{~m}$ Bed size $=1.8 \mathrm{~m}$

FIG. 1.-Field layout for tomato performance under trickle irrigation.

\section{RESULTS AND DISCUSSION}

Tables 1 and 2 indicate size arrangements of fresh harvested mature green tomatoes on three harvesting dates for the three water application rates (wet, moist and dry) in PS, NPS, PNS and NPNS plots, respectively. There was no significant difference at the $5 \%$ level among the 
TABLE 1.-Effect of varying water application rates, plastic mulch, staking on percentage of number of fresh harvested tomatoes (var. Walter) under drip irrigation. Date of transplanting: Nov 13, 1980

\begin{tabular}{|c|c|c|c|c|c|c|c|c|c|c|c|c|c|c|c|c|}
\hline \multirow{5}{*}{$\begin{array}{l}\text { USDA Fruit } \\
\text { Size Class }\end{array}$} & \multicolumn{16}{|c|}{ Number of Fruits $(\%)$} \\
\hline & \multicolumn{12}{|c|}{ Harvesting date } & \multirow{2}{*}{\multicolumn{4}{|c|}{ Seasonal average }} \\
\hline & \multicolumn{4}{|c|}{ Jan 19,1981} & \multicolumn{4}{|c|}{ Feb 2, 1981} & \multicolumn{4}{|c|}{ Feb 11, 1981} & & & & \\
\hline & \multicolumn{16}{|c|}{ Water application Treatments } \\
\hline & $\mathrm{T}^{2}{ }^{2}$ & $\mathrm{~T} 2$ & T3 & Avg. & $\mathrm{T} 1$ & T2 & T3 & Avg. & T1 & $\mathrm{T} 2$ & T3 & Avg. & $\mathrm{T} 1$ & T2 & T3 & Avg. \\
\hline \multicolumn{17}{|c|}{ No plastic mulch and nonstaked tomatoes (NPNS) } \\
\hline i & - & - & 1 & - & 一 & - & - & - & - & - & - & - & - & - & 1 & 1 \\
\hline 2 & 5 & 2 & 5 & 4 & 8 & 6 & 6 & 7 & 2 & 1 & 3 & 2 & 5 & 3 & 5 & 4 \\
\hline 3 & 7 & 3 & 5 & 5 & 7 & 8 & 7 & 7 & 2 & 1 & 6 & 3 & 5 & 4 & 6 & 5 \\
\hline 4 & 20 & 23 & 25 & 23 & 20 & 24 & 20 & 22 & 16 & 22 & 14 & 17 & 19 & 23 & 20 & 20 \\
\hline 5 & 28 & 23 & 29 & 26 & 32 & 23 & 27 & 27 & 22 & 16 & 23 & 20 & 27 & 21 & 25 & 24 \\
\hline 6 & 31 & 39 & 30 & 34 & 26 & 33 & 38 & 32 & 45 & 46 & 40 & 44 & 34 & 39 & 36 & 36 \\
\hline 7 & 8 & 8 & 5 & 7 & 7 & 6 & 2 & 5 & 12 & 11 & 13 & 12 & 9 & 8 & 7 & 8 \\
\hline 8 & 1 & 2 & - & 1 & - & - & - & - & 1 & 3 & 1 & 2 & 1 & 2 & - & 2 \\
\hline \multicolumn{17}{|c|}{ No plastic mulch and staked tomatoes (NPS) } \\
\hline 1 & - & 1 & 1 & 1 & 1 & 1 & - & 1 & - & - & - & - & 一 & 1 & - & 1 \\
\hline 2 & 3 & 1 & 6 & 3 & 3 & 10 & 3 & 5 & - & 1 & 1 & 1 & 2 & 4 & 3 & 3 \\
\hline 3 & 7 & 6 & 6 & 6 & 6 & 10 & 5 & 7 & 4 & 1 & 1 & 2 & 6 & 6 & 4 & 5 \\
\hline 4 & 26 & 28 & 22 & 25 & 28 & 24 & 17 & 24 & 13 & 17 & 10 & 10 & 22 & 23 & 17 & 20 \\
\hline 5 & 23 & 30 & 24 & 26 & 20 & 25 & 32 & 25 & 18 & 31 & 17 & 23 & 20 & 28 & 24 & 24 \\
\hline 6 & 36 & 29 & 31 & 32 & 33 & 29 & 34 & 32 & 48 & 39 & 48 & 46 & 39 & 32 & 38 & 36 \\
\hline 7 & 4 & 5 & 9 & 6 & 8 & 1 & 9 & 6 & 15 & 9 & 17 & 15 & 9 & 5 & 12 & 9 \\
\hline 8 & 1 & - & 1 & 1 & 1 & - & - & - & 2 & 2 & 6 & 3 & 2 & 1 & 2 & 2 \\
\hline \multicolumn{17}{|c|}{ Plastic mulch and nonstaked tomatoes (PNS) } \\
\hline 1 & 1 & 1 & 1 & 1 & 一 & - & - & - & - & - & - & - & - & - & - & - \\
\hline 2 & 7 & 7 & 5 & 6 & 7 & 7 & 4 & 6 & 1 & 3 & - & 1 & 5 & 6 & 3 & 5 \\
\hline 3 & 8 & 10 & 8 & 9 & 12 & 10 & 6 & 9 & 5 & 2 & 4 & 4 & 8 & 7 & 6 & 7 \\
\hline 4 & 37 & 29 & 28 & 31 & 25 & 24 & 25 & 25 & 20 & 13 & 16 & 16 & 27 & 22 & 24 & 24 \\
\hline 5 & 28 & 29 & 32 & 30 & 27 & 21 & 23 & 24 & 29 & 29 & 24 & 28 & 28 & 26 & 26 & 27 \\
\hline 6 & 17 & 21 & 25 & 21 & 22 & 31 & 35 & 29 & 29 & 43 & 49 & 40 & 23 & 32 & 36 & 30 \\
\hline 7 & 1 & 3 & 1 & 1 & 7 & 6 & 7 & 7 & 15 & 8 & 5 & 9 & 8 & 6 & 4 & 6 \\
\hline 8 & 1 & - & - & 1 & - & 1 & - & - & 1 & 2 & 2 & 2 & 1 & 1 & 1 & 1 \\
\hline \multicolumn{17}{|c|}{ Plastic mulch and staked tomatoes (PS) } \\
\hline 1 & 1 & - & 1 & 1 & 一 & - & 1 & - & - & 1 & - & - & 一 & 1 & 1 & 1 \\
\hline 2 & 8 & 3 & 12 & 7 & 8 & 3 & 5 & 5 & 5 & 1 & 2 & 3 & 7 & 2 & 7 & 5 \\
\hline 3 & 10 & 9 & 12 & 10 & 8 & 5 & 9 & 7 & 5 & 4 & 3 & 4 & 8 & 6 & 8 & 7 \\
\hline 4 & 31 & 31 & 29 & 30 & 29 & 16 & 27 & 25 & 23 & 13 & 19 & 18 & 27 & 20 & 24 & 24 \\
\hline 5 & 22 & 22 & 28 & 24 & 28 & 28 & 23 & 26 & 25 & 21 & 25 & 24 & 27 & 24 & 24 & 25 \\
\hline 6 & 26 & 29 & 18 & 24 & 26 & 39 & 8 & 31 & 32 & 47 & 44 & 41 & 27 & 38 & 30 & 31 \\
\hline 7 & 2 & 5 & 1 & 3 & 1 & 9 & 7 & 6 & 9 & 12 & 6 & 9 & 4 & 8 & 5 & 6 \\
\hline 8 & - & 1 & 1 & 1 & - & - & - & - & 1 & 1 & 1 & 1 & 一 & 1 & 1 & 1 \\
\hline
\end{tabular}

"USDA fruit size based on "USDA-tomatoes shipping point instructions."

${ }^{2} \mathrm{~T} 1, \mathrm{~T} 2, \mathrm{~T} 3=$ Water application based on tensiometer readings installed at 15,30 and $45 \mathrm{~cm}$ depth. 
TABLE 2.-Effect of varying water application rates, plastic mulch, staking on average weight (g) of fresh harvested tomatoes (var. Walter) under drip irrigation. Date of transplanting: Nov. 13, 1980.

\begin{tabular}{|c|c|c|c|c|c|c|c|c|c|c|c|c|c|c|c|c|}
\hline \multirow{5}{*}{$\begin{array}{l}\text { USDA Fruit } \\
\text { Size Class }\end{array}$} & \multicolumn{16}{|c|}{ Average weight of fresh harvested tomatoes, $\mathrm{g}$} \\
\hline & \multicolumn{12}{|c|}{ Harvesting date } & \multirow{2}{*}{\multicolumn{4}{|c|}{ Seasonal average }} \\
\hline & \multicolumn{4}{|c|}{ January 19,1981} & \multicolumn{4}{|c|}{ February 2, 1981} & \multicolumn{4}{|c|}{ February 11,1981} & & & & \\
\hline & \multicolumn{16}{|c|}{ Water application treatments. $(\mathrm{T} 1=$ wet, $\mathrm{T} 2=$ moist, $\mathrm{T} 3=\mathrm{dry})$} \\
\hline & T1 & $\mathrm{T} 2$ & T3 & Avg. & T1 & $\mathrm{T} 2$ & T3 & Avg. & T1. & T2 & $\mathrm{T} 3$ & Avg. & $\mathrm{T} 1$ & $\mathrm{~T} 2$ & T3 & Avg. \\
\hline \multicolumn{17}{|c|}{ No plastic mulch and nonstaked tomatoes (NPNS) } \\
\hline 1 & - & - & 211.6 & 211.6 & - & - & - & - & - & - & - & - & - & - & - & - \\
\hline 2 & 182.0 & 191.6 & 177.1 & 183.6 & 191.7 & 199.0 & 200.1 & 196.9 & 172.7 & 156.1 & 176.8 & 168.5 & 182.1 & 182.2 & 184.7 & 183.0 \\
\hline 3 & 156.1 & 164.2 & 153.9 & 158.1 & 163.8 & 169.3 & 171.4 & 168. & 149.8 & 169.8 & 165.4 & 161.7 & 156.6 & 167.8 & 163.6 & 162.7 \\
\hline 4 & 136.0 & 138.5 & 139.6 & 138.0 & 142.1 & 148.1 & 141.6 & 143.9 & 138.3 & 135.9 & 136.6 & 136.9 & 138.8 & 140.8 & 139.3 & 139.6 \\
\hline 5 & 116.9 & 119.2 & 119.1 & 118.4 & 120.9 & 122.8 & 125.4 & 123.0 & 177.7 & 120.3 & 128.1 & 142.0 & 138.5 & 120.8 & 124.2 & 127.8 \\
\hline 6 & 97.3 & 99.8 & 97.9 & 98.3 & 102.1 & 104.8 & 101.1 & 102.7 & 95.5 & 94.3 & 97.9 & 95.9 & 98.3 & 99.6 & 98.9 & 98.9 \\
\hline 7 & 75.7 & 78.3 & 79.1 & 77.7 & 77.2 & 78.5 & 76.0 & 77.2 & 74.4 & 78.1 & 73.0 & 75.2 & 75.8 & 78.3 & 76.0 & 76.7 \\
\hline 8 & 65.6 & 60.1 & - & 62.8 & - & - & - & - & 54.6 & 55.1 & 57.1 & 55.6 & 60.1 & 57.6 & 57.1 & 58.3 \\
\hline Avg. & 118.5 & 121.7 & 139.8 & 126.7 & 132.9 & 137.1 & 135.9 & 135.3 & 123.3 & 115.7 & 119.3 & 119.4 & 124.9 & 124.8 & 131.7 & 127.1 \\
\hline \multicolumn{17}{|c|}{ No plastic mulch and staked tomatoes (NPS) } \\
\hline 1 & - & 240.5 & 249.7 & 245.1 & 250.5 & 181.8 & - & 216.2 & - & - & - & - & 250.5 & 211.2 & 249.7 & 237.1 \\
\hline 2 & 170.4 & 160.5 & 198.8 & 176.6 & 188.2 & 183.9 & 178.8 & 183.6 & - & 181.5 & 163.4 & 172.5 & 179.3 & 175.3 & 180.3 & 178.3 \\
\hline 3 & 162.2 & 153.3 & 163.1 & 159.5 & 151.2 & 165.8 & 159.4 & 158.8 & 167.1 & 162.3 & 173.5 & 167.6 & 160.2 & 160.5 & 165.3 & 162.0 \\
\hline 4 & 144.5 & 140.0 & 142.3 & 142.3 & 142.9 & 142.1 & 145.7 & 143.6 & 144.9 & 137.3 & 139.6 & 140.6 & 144.1 & 139.8 & 142.5 & 142.1 \\
\hline 5 & 122.8 & 117.5 & 122.8 & 121.0 & 127.7 & 124.1 & 120.5 & 124.1 & 120.9 & 116.7 & 118.6 & 118.7 & 123.8 & 119.4 & 120.6 & 121.3 \\
\hline 6 & 102.8 & 96.9 & 98.5 & 99.4 & 103.1 & 101.4 & 101.5 & 102.0 & 99.6 & 95.3 & 98.3 & 97.7 & 101.8 & 97.9 & 99.4 & 99.7 \\
\hline 7 & 72.5 & 71.8 & 74.8 & 73.0 & 88.7 & 81.7 & 84.1 & 84.8 & 73.3 & 70.6 & 71.2 & 71.7 & 73.0 & 74.7 & 76.7 & 74.8 \\
\hline 8 & 72.9 & - & 73.5 & 73.2 & 55.1 & - & - & 55.1 & 53.3 & 49.4 & 53.7 & 52.1 & 60.4 & 49.4 & 63.6 & 57.8 \\
\hline Avg. & 121.2 & 140.1 & 140.4 & 133.9 & 138.4 & 140.1 & 131.7 & 136.7 & 109.8 & 116.2 & 116.9 & 114.3 & 123.1 & 132.1 & 129.7 & 128.3 \\
\hline
\end{tabular}




\begin{tabular}{|c|c|c|c|c|c|c|c|c|c|c|c|c|c|c|c|c|}
\hline \multicolumn{17}{|c|}{ Plastic mulch and nonstaked tomatoes (PNS) } \\
\hline 1 & 203.9 & 206.7 & 212.0 & 207.5 & - & 一 & - & 一 & - & - & - & - & 203.9 & 206.7 & 212.0 & 207.5 \\
\hline 2 & 176.5 & 180.7 & 187.3 & 181.5 & 193.0 & 187.7 & 206.5 & 195.7 & 160.8 & 179.5 & - & 170.2 & 176.8 & 182.6 & 191.1 & 183.5 \\
\hline 3 & 160.3 & 161.1 & 165.2 & 162.2 & 170.9 & 161.6 & 160.9 & 164.5 & 164.6 & 161.6 & 157.7 & 161.3 & 165.3 & 161.4 & 161.3 & 162.7 \\
\hline 4 & 138.6 & 136.3 & 142.4 & 139.1 & 145.9 & 140.4 & 142.5 & 142.9 & 138.9 & 136.9 & 136.9 & 137.6 & 141.1 & 137.9 & 140.9 & 139.9 \\
\hline 5 & 117.7 & 119.9 & 121.4 & 119.7 & 123.9 & 120.4 & 119.8 & 121.4 & 123.2 & 115.5 & 121.4 & 120.0 & 121.6 & 118.6 & 120.8 & 120.3 \\
\hline 6 & 102.8 & 100.2 & 101.9 & 101.6 & 99.8 & 108.2 & 103.5 & 103.8 & 102.1 & 95.3 & 99.8 & 99.1 & 101.6 & 101.2 & 102.2 & 101.7 \\
\hline 7 & 83.7 & 83.7 & 78.0 & 81.8 & 82.1 & 76.6 & 75.6 & 78.1 & 78.1 & 75.0 & 75.1 & 76.1 & 81.3 & 78.4 & 76.2 & 78.6 \\
\hline 8 & 77.2 & 一 & - & 77.2 & - & 64.5 & - & 64.5 & 57.9 & 66.6 & 50.1 & 58.2 & 67.6 & 65.6 & 50.1 & 61.1 \\
\hline Avg. & 132.6 & 141.2 & 144.0 & 139.3 & 135.9 & 122.8 & 134.8 & 131.2 & 117.9 & 118.6 & 106.8 & 114.4 & 128.8 & 127.5 & 128.5 & 128.3 \\
\hline \multicolumn{17}{|c|}{ Plastic mulch and staked tomatoes (PS) } \\
\hline 1 & 247.4 & - & 226.9 & 237.2 & - & - & 185.9 & 185.9 & - & 222.4 & 一 & 222.4 & 247.4 & 222.4 & 206.4 & 225.4 \\
\hline 2 & 187.2 & 181.2 & 182.9 & 183.8 & 186.1 & 188.0 & 175.3 & 183.1 & 159.7 & 175.9 & 180.2 & 171.9 & 177.7 & 181.7 & 179.5 & 179.6 \\
\hline 3 & 163.5 & 167.3 & 169.8 & 166.9 & 165.1 & 171.2 & 167.5 & 167.9 & 164.6 & 154.4 & 177.6 & 165.6 & 164.4 & 164.3 & 171.6 & 166.8 \\
\hline 4 & 145.1 & 142.0 & 143.8 & 143.6 & 144.1 & 146.7 & 138.5 & 143.1 & 141.1 & 137.4 & 146.1 & 141.5 & 143.4 & 142.0 & 142.8 & 142.7 \\
\hline 5 & 123.5 & 123.6 & 119.2 & 122.1 & 122.5 & 126.8 & 122.2 & 123.8 & 117.6 & 112.7 & 117.6 & 115.9 & 121.2 & 121.0 & 119.7 & 120.6 \\
\hline 6 & 106.4 & 104.2 & 104.2 & 104.9 & 100.7 & 106.1 & 100.4 & 102.4 & 93.1 & 90.7 & 99.8 & 94.5 & 100.1 & 100.3 & 101.5 & 100.6 \\
\hline 7 & 87.1 & 85.9 & 78.4 & 83.8 & 70.8 & 78.5 & 80.7 & 76.7 & 74.1 & 70.9 & 75.4 & 73.5 & 77.3 & 78.4 & 78.2 & 77.9 \\
\hline 8 & - & 64.1 & 74.9 & 69.5 & - & - & 一 & - & 63.9 & 38.5 & 60.1 & 54.2 & 63.9 & 51.3 & 67.5 & 60.9 \\
\hline Avg. & 151.5 & 124.0 & 137.5 & 137.7 & 131.6 & 136.2 & 138.6 & 135.5 & 116.3 & 125.4 & 122.4 & 121.4 & 133.1 & 128.5 & 132.8 & 131.5 \\
\hline
\end{tabular}


TABLE 3.-Effect of varying water application rates on percentages of fruit quality of tomatoes (var. Walter) under drip irrigation. Date of transplanting: Nov. 13, 1980

\begin{tabular}{|c|c|c|c|c|c|c|c|c|c|}
\hline \multirow{4}{*}{$\begin{array}{l}\text { Sr. } \\
\text { No. }\end{array}$} & \multirow{4}{*}{$\begin{array}{l}\text { Treat- } \\
\text { ment }\end{array}$} & \multicolumn{8}{|c|}{ Subtreatments $^{1}$} \\
\hline & & \multicolumn{2}{|c|}{ NPNS } & \multicolumn{2}{|c|}{ NPS } & \multicolumn{2}{|c|}{ PNS } & \multicolumn{2}{|c|}{ PS } \\
\hline & & \multicolumn{8}{|c|}{ Average percentage distribution ${ }^{2}, \%$} \\
\hline & & $\begin{array}{l}\text { Defective } \\
\text { tomatoes }\end{array}$ & $\begin{array}{l}\text { U.S. Grade I } \\
\text { tomatoes }\end{array}$ & $\begin{array}{l}\text { Defective } \\
\text { tomatoes }\end{array}$ & $\begin{array}{c}\text { U.S. Grade I } \\
\text { tomatoes }\end{array}$ & $\begin{array}{l}\text { Defective } \\
\text { tomatoes }\end{array}$ & $\begin{array}{c}\text { U.S. Grade I } \\
\text { tomatoes }\end{array}$ & $\begin{array}{l}\text { Defective } \\
\text { tomatoes }\end{array}$ & $\begin{array}{c}\text { U.S. Grade I } \\
\text { tomatoes }\end{array}$ \\
\hline \multicolumn{10}{|c|}{ Jan. 19,81} \\
\hline 1 & $\mathrm{~T} 1$ & 31.7 & 68.3 & 26.4 & 73.6 & 24.7 & 75.3 & 29.0 & 71.0 \\
\hline 2 & $\mathrm{~T} 2$ & 23.0 & 77.0 & 25.0 & 75.0 & 26.0 & 74.0 & 33.4 & 66.6 \\
\hline 3 & $\mathrm{~T} 3$ & 27.4 & 72.6 & 23.7 & 76.3 & 29.0 & 71.0 & 29.4 & 70.6 \\
\hline \multicolumn{10}{|c|}{ Feb. 2, 81} \\
\hline 4 & $\mathrm{~T} 1$ & 48.0 & 52.0 & 46.7 & 53.3 & 36.0 & 64.0 & 46.0 & 54.0 \\
\hline 5 & $\mathrm{~T} 2$ & 42.4 & 57.6 & 41.0 & 59.0 & 42.4 & 57.6 & 42.7 & 57.3 \\
\hline 6 & T3 & 36.7 & 63.3 & 41.7 & 58.3 & 44.4 & 55.6 & 42.0 & 58.0 \\
\hline \multicolumn{10}{|c|}{ Feb. 11, 81} \\
\hline 7 & $\mathrm{~T} 1$ & 59.0 & 41.0 & 54.4 & 45.6 & 52.7 & 47.3 & 52.0 & 48.0 \\
\hline 8 & $\mathrm{~T} 2$ & 57.4 & 42.6 & 51.4 & 48.6 & 52.4 & 47.6 & 57.0 & 43.0 \\
\hline 9 & $\mathrm{~T} 3$ & 51.0 & 49.0 & 59.6 & 40.4 & 52.4 & 47.6 & 48.7 & 51.3 \\
\hline \multicolumn{10}{|c|}{ Seasonal } \\
\hline 10 & $\mathrm{~T} 1$ & 46.3 & 53.7 & 42.5 & 57.5 & 37.8 & 62.2 & 42.4 & 57.6 \\
\hline 11 & $\mathrm{~T} 2$ & 41.0 & 59.0 & 39.2 & 60.8 & 40.3 & 59.7 & 44.4 & 55.6 \\
\hline 12 & $\mathrm{~T} 3$ & 38.4 & 61.6 & 41.7 & 58.3 & 42.0 & 58.0 & 40.1 & 59.9 \\
\hline
\end{tabular}

${ }^{1}$ NPNS $=$ No plastic-no stakes; NPS = No plastic-stakes; PNS = Plastic-no stakes; PS = Plastic-stakes.

${ }^{2}$ Based on 6 replicates of 50 mature green fruits each. 
subtreatments (PS, PNS, NPNS and NPS) and main treatments (T1, T2, T3) in any sizes, except sizes 2, 4, 6 on Jan. 19; size 2 on Feb. 2; and sizes 2 and 6 on Feb. 11, 1981 on weight basis, respectively. On number basis, there was no significant difference at the 5\% level in any sizes except sizes 2 to 7 on Jan. 19; and sizes 2 and 8 on Feb. 11, 1981, respectively. Table 1 shows that percentage of number of fruits was highest in size 6 for all treatments and subtreatments. The average fruit weight was highest during the second picking for all treatment in the NPNS, NPS and PNS plots. In the PS plots, the first picking gave the highest fruit weight compared with that of second and third pickings. Seasonal mean fruit weight in the T1, T2, T3 treatments was 124.9, 124.8

TABLE 4.-Effect of varying water application rates on vitamin A content of tomatoes under drip irrigation. Date of transplanting: Nov. 13, 1980. Date of observation: Feb. 11, 1981

\begin{tabular}{ccc}
\hline \multirow{2}{*}{ Treatment $^{1}$} & Subtreatment $^{2}$ & Vitamin A (U. I.) $^{2}$ \\
\cline { 2 - 2 } T1 $=$ Wet & NPNS & $450 \mathrm{~nm}$ \\
& NPS & 1204.5 \\
& PS & 797.3 \\
T2 $=$ Moist & PNS & 699.4 \\
& NPNS & 1114.9 \\
& NPS & 1577.6 \\
T3 $=$ Dry & PS & 903.4 \\
& PNS & 820.0 \\
& NPNS & 1521.9 \\
& NPS & 1108.4 \\
& PS & 797.5 \\
& PNS & 1141.3 \\
\hline
\end{tabular}

${ }^{1} \mathrm{~T} 1, \mathrm{~T} 2, \mathrm{~T} 3=$ Water application rates were based on readings of tensiometers installed at 15,30 and $40 \mathrm{~cm}$ depth.

${ }^{2}$ NPNS $=$ No plastic-no stakes; NPS = No plastic-stakes; PS = Plastic-stakes; PNS = Plastic-no stakes.

and $131.7 \mathrm{~g}$ in NPNS plots; 123.1, 132.1 and 129.7 in NPS plots; 128.8 , 127.5 and 128.5 in PNS plots; and 133.1, 128.5 and 132.8 in PS plots, respectively, as indicated in table 2.

Table 3 reveals effects of varying water application rates, plastic mulch and staking on average percentage distribution of US grade I and defective tomatoes. There was no significant difference at the 5\% level among the subtreatments and treatments. The dry treatment gave the highest percentage of US grade I tomatoes in NPNS and PS plots compared with that of the moist treatment in the NPS and the wet treatment in the PNS plots, respectively. Table 4 indicates effects of these treatments on vitamin A content in tomatoes picked Feb. 11, 1981. The vitamin A 
content was highest in NPNS plots of the wet and moist treatment, and in PS plots of the dry treatment. PS plots in the wet and moist treatments, and NPS plots in the dry treatment gave lowest values of vitamin A content.

\section{RESUMEN}

La distribución y el porcentaje portamanos de tomates bien desarrollados pero sin pintonear y el contenido de vitamina A se evaluaron en tomates cultivados con cubierta plástica y con estacas (PS), con cubierta plástica sin estacas (PNS), sin cubierta plástica y con estacas (NPS), y sin cubierta plástica y sin estacas (NPNS) regados por goteo en tratamientos mojado, húmedo y seco, respectivamente. En la distribución por tamaños, el USDA \#6 arrojó el porcentaje más alto en todos los tratamientos y subtratamientos. En todos los tratamientos el peso medio de las frutas fue mayor en la segunda cogida en las parcelas NPNS, NPS y PNS comparado con el de la primera en los predios PS. El tratamiento seco rindió el porcentaje más alto de tomates "U.S. grade I" (calidad \#1) en las parcelas NPNS y PS. El contenido de vitamina A fue más alto en los tomates de las parcelas NPNS en los tratamientos mojado y húmedo; y en el predio PS en los tratamientos secos. Los tomates de las parcelas PS en los tratamientos mojado y húmedo; y los de las NPS en el tratamiento seco arrojaron los valores más bajos de vitamina $\mathrm{A}$. 